\title{
Fragility evaluation of Tehran traffic control cameras during earthquakes and the introduction of dynamic emergency paths toward a sustainable city
}

\author{
H. Behruz ${ }^{1}$, A. Lavasani $\operatorname{rad}^{1}$, M. Hosseini ${ }^{2}$ \& A. P. Chavoshy ${ }^{1}$ \\ ${ }^{1}$ Tehran Traffic Control Company, Iran \\ ${ }^{2}$ International Institute of Earthquake Engineering and Seismology, Iran
}

\begin{abstract}
At the time of an earthquake and after, maintaining the performance of the vital systems of the cities (roads, gas, electricity, water and sanitation, and telecommunications) - lifelines - is top priority. Among them, the transportation network appears to be the most vital for emergency aid. Routes for emergency relief services are statistically selected by predicting earthquake damage to roads and various components of the surrounding structures, but this prediction becomes unrealistic due to the lack of documentation of the components listed above.

Tehran is now equipped with a network of more than 1000 surveillance cameras that covers the most critical parts of the road network and if they remain functional after the earthquake, they will potentially become one of the most important management tools for crisis management and identification of emergency routes.

This research evaluates the ability of Tehran's lifelines and especially road network surveillance cameras to maintain their functionality after an earthquake, and also provides suggestions for organizing systems of risk management and introduces a dynamic emergency routes concept.

Keywords: sustainable city, earthquake, Tehran, control camera, emergency routes.
\end{abstract}




\section{Introduction}

The last earthquake in Tehran measuring 7.2 on the Richter scale was recorded in 1830. Considering there are 7 important fault lines in Tehran and the history of earthquakes occurring in the city, it is likely that a big earthquake will occur in Tehran in the future $[1,2]$.

The systems of supplying electrical energy, gas and oil, as well as water and wastewater disposal, transportation, information and telecommunication, called lifelines, are those structures that have a critical role in city performance and their damage will result in serious losses in rescuing and saving the wounded and injured in an earthquake. Considering the density of lifelines in Tehran and the lack of resistance of many of them against an earthquake, if an earthquake happened in Tehran, we must expect several interruptions in water, wastewater and gas systems, the fall of some pylons, collapse of some bridges and similar accidents; and extensive fire may occur as a result.

Multiple studies show that the lifelines of the city of Tehran are not ready against the earthquake which will surely happen [3-5]. Which strategies should be adopted to improve current conditions?

\section{Current conditions of urban transportation and the road system in Tehran}

It is obvious that among all the lifelines, the transportation system plays the main role during and after an earthquake. The city of Tehran struggles against all types of difficulties and disorders of traffic on ordinary days. In the event of an earthquake, almost all the vehicles moving on routes, which are still capable to be used, change direction to reach relatives. The vehicles that start to move will then be added to. Moreover, the blocking of moving ways due to damaged or abandoned vehicles, falling or displacement of all types of road equipment, damage and falling of existing structures, such as bridges, rubble of buildings, trees as well as temporary aid gatherings is easily predictable. If they do not obstruct ways, they will still cause considerable reduction in the roadway capacity. In addition, the road structure itself, may be seriously damaged causing the route to go out of service. Thus it is predicted that a major part of the road system will become useless after an earthquake and the few that will be operable will be some parts of the highways and perhaps arterials.

Like other lifelines mentioned earlier, the road system can be divided into different levels of vulnerability, and can be modelled for evaluation of their likely damage. Considering the old masonry buildings in the south part of the city, on the one hand, and prediction of more structural damage in that area because of alluvial texture of its soil, on the other, as well as a high percentage of local access streets (lower width and more interconnection), the road system will certainly have less operability.

Naturally, the amount of damage mostly depends on the intensity of the earthquake and should be applied in modelling as different scenarios. 


\section{Challenge of rate of reliability of prediction in determination of emergency routes}

Crisis arising factors resulting from the occurrence of an earthquake for the local transportation system of Tehran can be categorized as follows [6]:

I. Damage to bridges, numbering approximately 250 cases, and in the case of a severe earthquake, this can block two major routes.

II. Instability of slopes adjacent to many highways that can partly or completely block routes.

III. Collapse of high-rise buildings beside highways and many main streets that can completely block the route and/or at least make it unsafe.

IV. Damage to water, gas and wastewater systems will not only threaten streets and highways, but also subway tunnels.

V. Fall of pylons and BTSs, in addition to blocking the routes, increases the possibility of fire.

VI. Abandonment of vehicles in the streets by the drivers due to an impossibility to move and their intention to access their houses and relatives

VII. Breakdown of traffic lights due to power outage that intensify the disorder resulting from the crisis of earthquake.

Many of the mentioned cases cannot be easily predicted, making analysis simulation models necessary.

\subsection{Looking at information}

The emergency system of Tehran is mostly comprised of highways and an arterial network with the length of $374 \mathrm{~km}$ and $299 \mathrm{~km}$ that respectively forms $14 \%$ and $11 \%$ of the routes in Tehran. With a view to emergency access, scattering heterogeneity of such network makes the centre of the city an impenetrable area.

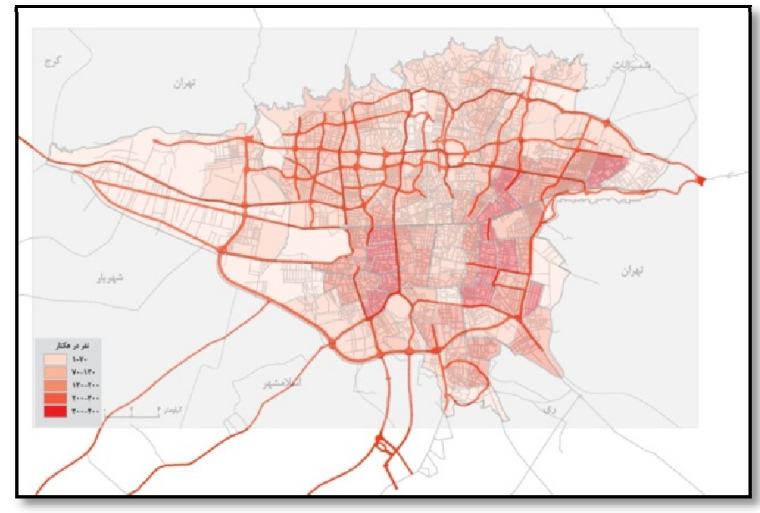

Figure 1: Location of highway network of the city of Tehran in a population density map (bolder is more condensed). 
The latest information shows that density of the population of such an area is very high; especially during the day when it attracts many official and commercial travellers (Figure 1).

In addition, the density of health centres in that area is more than in other urban areas and access to them is a serious challenge (Figure 2).

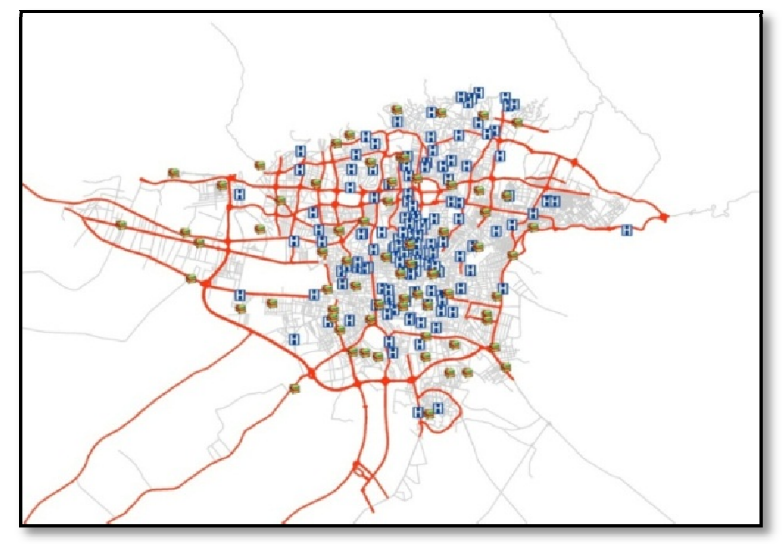

Figure 2: $\quad$ Location of hospitals $(\mathrm{H})$ and fire departments.

There are 250 roadway bridges and 732 pedestrian bridges in Tehran. Most bridges are naturally located on a highway system and therefore serious damage to them causes problems for the performance of the emergency network (Figure 3).

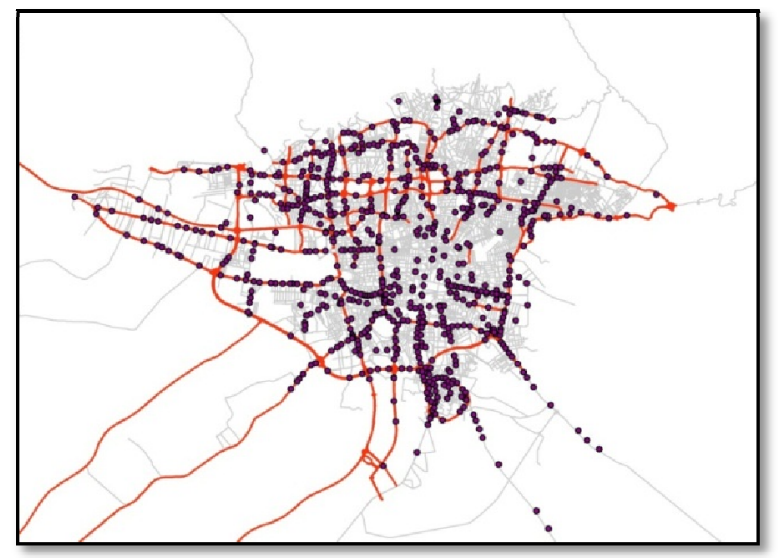

Figure 3: $\quad$ Location of pedestrian bridges.

Figure 4 shows the aid stations based on Japan International Cooperation Agency's (JICA) studies. Unlike the previous items, considerable numbers of 
such stations are located within the low influenced limit and require less special investigation to establish necessary access.

As mentioned above, very comprehensive planning is required to providing access of aid forces to the populated centres, health centres as well as rescue centres during the earthquake. Nevertheless, planning depends on predictions of the rate of damage to structures such as bridges and buildings on the margins of the emergency routes (and therefore the rate of operability of routes) and/or embankment slopes, which is not easily achievable.

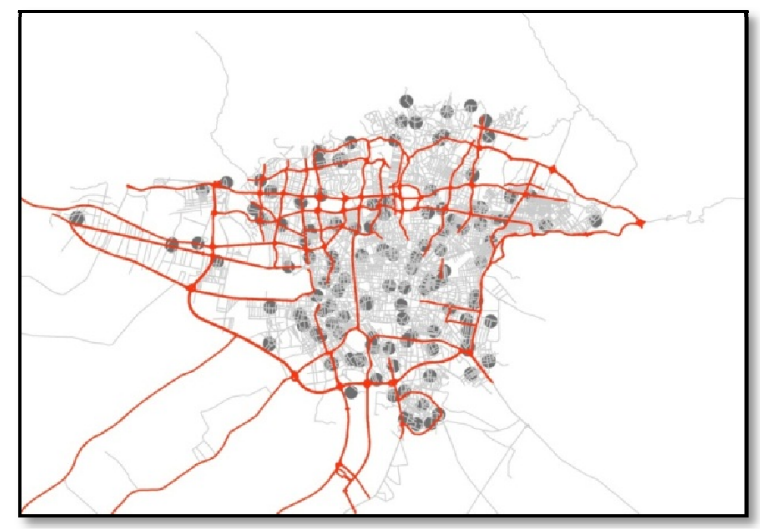

Figure 4: $\quad$ Scattering of aid stations based on JICA studies.

Executive weaknesses, impossibility to diagnose the weak points of existing structures, high approximation in the prediction of failure mechanisms, high approximation in prediction of rate of decrease in roadway capacity due to the collapse of bridges or buildings, impossibility to predict the behaviour of drivers after the earthquake, approximation in the general prediction of damage and similar factors, extremely reduce the reliability of programs for the management of emergency routes.

\section{Utilization of traffic control equipment in crisis management and determination of emergency routes}

Among the equipment of Tehran traffic management, a systematic structure of 1000 video surveillance cameras can be used to provide a timely correct estimation of damage to lifelines after the occurrence of an earthquake. Web kiosks, which are currently used in specific bus stations for two-way communication of passengers for determination of optimum route, can be completed by software programming as a two-way communication device for people. For instance, the people can use it to insert their name in the database where they are situated for information of the relatives seeking them. 


\subsection{Video surveillance cameras}

The crisis management centres can be connected to an accurate system of video surveillance cameras for management purposes by seeing damage as well as the manner of aiding (relief). In such cases:

I. The physical stability of the camera network after the earthquake should be guaranteed.

II. The physical stability of camera control and crisis management centres should be confirmed.

III. The cameras' network and monitoring centres should maintain their usefulness after the earthquake and this includes the stability of all connections as well as communication devices as components of the networks (such as fibre optics).

IV. Support centres for monitoring as well as repair and design services should be ready to work.

V. The manner of aiding and using emergency routes instead of relying on static models should be planned so as to have the ability to provide services dynamically and send aid groups if necessary (based on the decision of the control centre) to required areas determined by monitoring.

VI. Two-way communication of aid stations and monitoring centres, preferably online, and permanent communication between mobile groups and the monitoring centres and aid stations with minimum deficiency, should be ensured.

Currently, more than 1000 video surveillance cameras cover the road network of Tehran. Figure 5 shows that most networks used as the emergency network in Tehran after the crisis will be covered by such cameras; and therefore, valuable information of the rate of damage to the network and determination of likely substituting routes, after the earthquake, will be provided. It is known that there are considerable numbers of video surveillance cameras in the central area of the

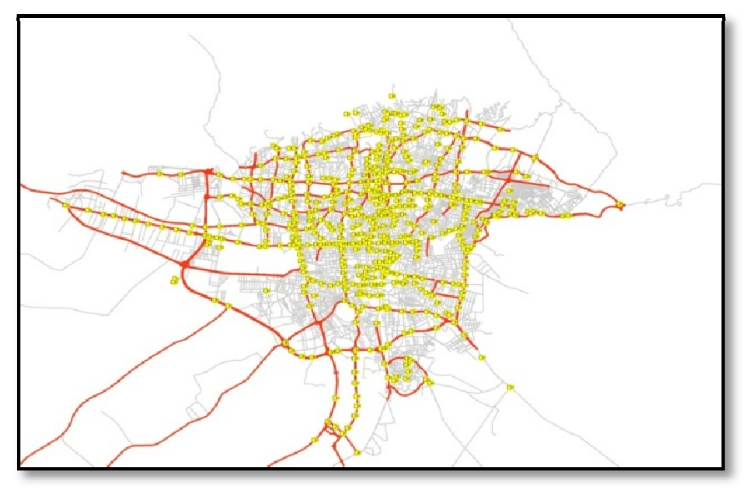

Figure 5: $\quad$ Location of video surveillance cameras. 
city, where there is the concentration of health centres and lack of access to the highway network, which can handle optimum management of rescue facilities in such a critical area (Figure 5).

It is necessary to consider this fact that the points of Figure 5, considering the high resolution of cameras, covers a more extensive area and good quality of pictures increases the advantages of this method. Although, the south part of the city benefits from less cover, such weakness will be covered to some extent by the possibility of aiding such areas from outside of the city and the south side of Tehran. Figure 6 shows a sample of video cover of such cameras [8].

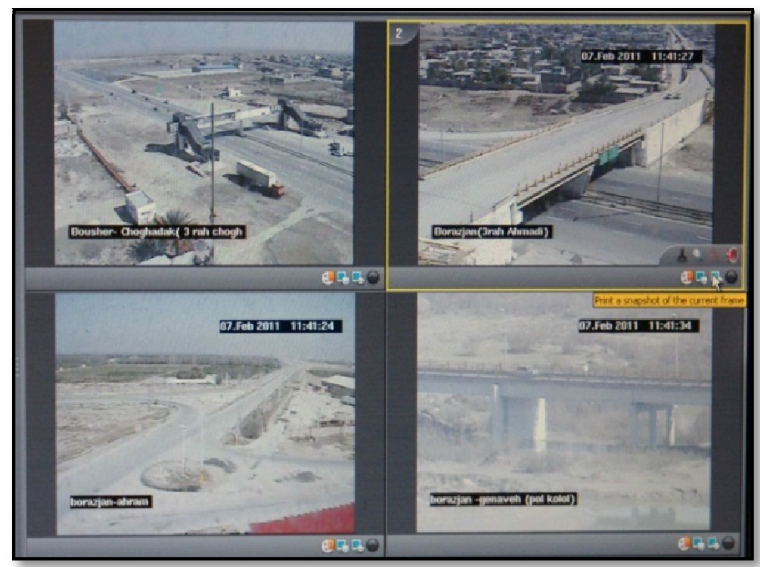

Figure 6: Control panel of video surveillance cameras and quality pictures of structures.

In the next part of this study, stability of the cameras and their monitoring centres as well as the fibre optics connecting them, and wireless network and power supply of the network during the earthquake will be assessed.

\section{Stability and usefulness of equipment at the time and after the occurrence of an earthquake}

The stages of studying the rate of usefulness for the equipment are as follows:

I. Stability of equipment structure;

II. Communication platform stability (wireless, cable or fibre optics and their equipment);

III. Stability of connections;

IV. Stability of control centres;

V. Electrical grid stability;

VI. Ability of the aiding system to use this equipment (including necessary training);

VII. Survival of repairing system after the earthquake. 


\subsection{Stability of equipment structure}

Video surveillance cameras are assembled on masts of 6 to 20 meters height with an octagonal and changeable section. In order to control the stability of the structure, SAP software is used, while controlling the stability of foundation (i.e. slippage and overturning control) is done manually due to simplicity of the structure.

According to displacement, the structure shows complete stability against the earthquake being introduced to the modelled structure for Tehran city. Designing documents of structure shows the dominance of input wind force over the mast, so the structure is calculated for wind force which is much more than the amount calculated for an earthquake. Therefore, structure destruction against earthquake does not seem probable.

Meanwhile, the foundation of structure with dimensions of $1.5 \times 2.2 \times 2.2$ has a weight equivalent to 18.15 tons, and whereas at the time of designing, the structure has been controlled for overturning due to wind (dominant over earthquake) and it is completely resistant, therefore, there would be no need to control for overturning due to an earthquake. In a similar way, connections' screws and also pillars' plate are calculated and controlled for movement due to wind; therefore, it will have enough stability against an earthquake. The total weight of the camera and set of equipment assembled at the tip of the mast is less than $20 \mathrm{~kg}$. Considering the low centralized mass modelling of this mass at the tip of the structure it would be useful for controlling deformations, but there would be no fear of destruction of structure [7].

Control of foundation slippage which is another necessary control is not of concern, because the structure is buried and also due to the great weight of the foundation which is calculated for forces larger than wind.

\subsection{Communication platform stability}

The communication platforms of most cameras are of fibre optics. The studies show that concrete pools have been embedded in specified distances, inside of which there are rings of fibre in order to obtain the required length (of fibres) (Figure 9).

This kind of mechanism would help to extend to the required length at the time of movements and deformations resulting from dragging, and prevent disconnections.

Wireless connections are of short-range, which has little sensitivity towards earthquake damage.

\subsection{Stability of connections}

During the years of operation, connections of cameras show adequate stability against wind movements. However, structures including connection equipment such as TDP and POE cabinets may be modelled for evaluating destruction mechanisms (Figure 10). 


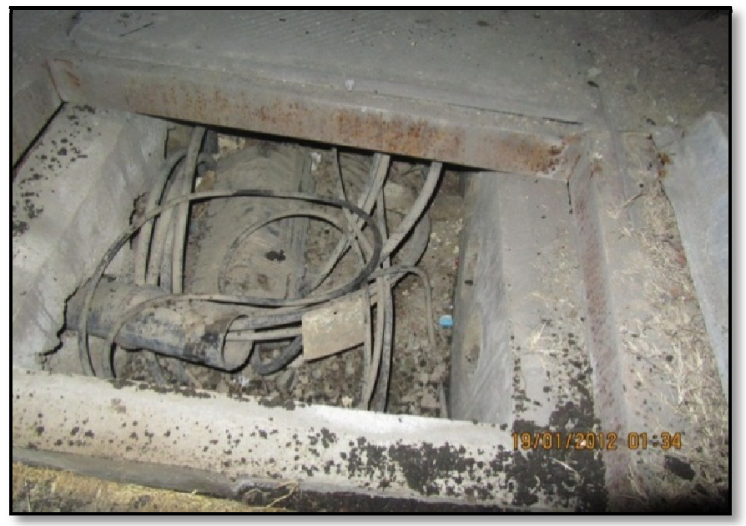

Figure 7: $\quad$ Fibre optics and added length cables controlling pool.

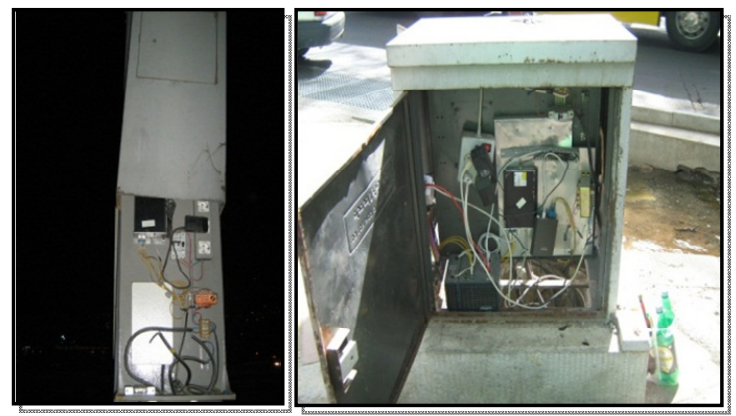

Figure 8: $\quad$ Connection equipment (POE and TDP) at the foot of the masts.

\subsection{Stability of control centres}

Present structures of control centres have no faults or weaknesses in design as they have been built recently according to new standards. Although, from the point of view of execution, precise controls and also extensive studies for controlling the local stability of assembled equipment in these centres (such as monitors assembled on walls) are necessary. Designers should consider special arrangements for local stability of such equipment in the structures to be built from now on.

\subsection{Electrical grid stability}

Inconsistency of the electrical grid to cameras is one of the serious concerns for their operation after the occurrence of an earthquake. Most connections available in masts' cabinets are of normal type (plug and socket) and are a significant factor in the cameras being out of service in normal conditions, based on the repair and maintenance reports. Sometimes, slight shakes at the time of repairing result in these plugs being removed from sockets, and therefore, may be a reason 
for serious concern if disconnecting the cameras from the network at the time of an earthquake.

\section{Suggestions for improving traffic management and control system with the approach of crisis management}

As previously studied, a static method for determining emergency routes in urban transportation network at the time of an earthquake is on the basis of estimates gained from different areas such as the extent of damage incurred to buildings and bridges, and the effect of this damage on the efficiency of emergency routes. Also, it has been evident, that due to various reasons, these estimates may be far from reality, in which case a large part of efficiency of rescue system will be affected. Therefore, it is suggested that part of planning is allocated to a dynamic system for rescuing in which certain parts of the system have the capability of rapid local displacement (human and rescuing equipment) on the basis of guidance of crisis control centres which are using the power of traffic control and management equipment such as cameras. For improvement of the existing conditions the following suggestions are offered:

I. Removing the problems caused by design weaknesses of the electrical part of cameras (on the basis of stability against an earthquake), and considering all necessary measures. For example, as it is shown in Figure 12, loose electrical connections may cause serious problems at the time of an earthquake.

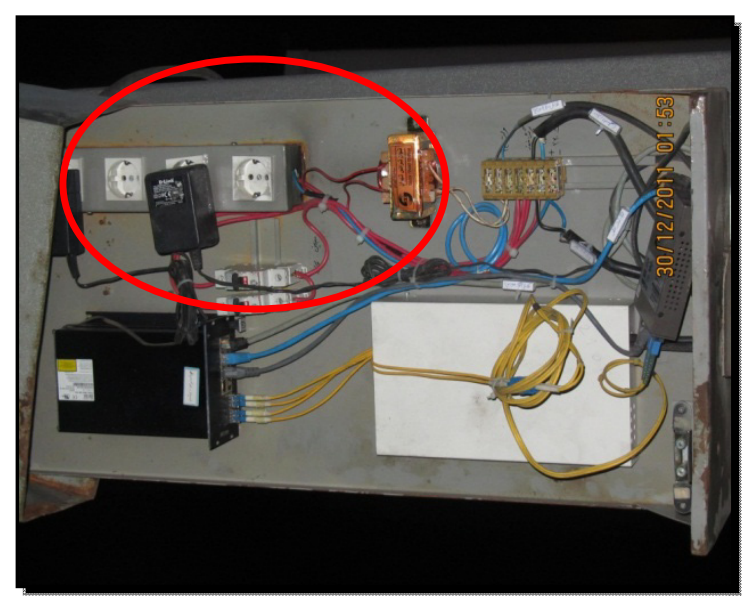

Figure 9: Instability of electrical connections against vibrations and shakes caused by an earthquake.

Also, creating an emergency power grid for supplying the cameras is among the priorities to be considered.

II. Completing video surveillance cameras network on the basis of crisis management needs. 
III. Reviewing the structure of the crisis management system on the basis of using the maximum ability of cameras.

IV. Reviewing the structures of determining emergency routes and defining dynamic routes specified on the basis of online data.

V. Constructing video surveillance centres with the approach of crisis management against severe earthquakes.

VI. Definition of lifelines' management accessibilities and the manner of their interaction at the time of earthquake.

VII. Furnishing bases and headquarters with facilities in order to access the information needed from dynamic emergency routes network.

VIII. Study of camera connections, communication routes (fibre optic and wireless) as to the stability against earthquakes and removing design and execution problems.

IX. Definition of emergency repairing system for overcoming the damage incurred to the video surveillance network at the time of an earthquake.

\section{Conclusion}

This study shows that:

I. It is not exactly clear that regarding efficiency of emergency routes after earthquake, to what extent would they be in accordance with anticipations.

II. With the help of more than 1000 video surveillance cameras of Tehran city as an effective tool in determining the routes surviving after an earthquake, and also determining the routes which are closed or opened during relief (rescuing) operations, online actions can be taken for identifying routes in order to get access to population centres, relief (rescuing) operation centres, etc.

III. Special relief (rescue) workers should be necessarily trained for rendering dynamic rescue operations and should be also furnished with mobile equipment so that in emergencies like earthquakes they could come to help and assist the injured.

IV. Although the stability of the camera network and preservation of its efficiency has been studied and it has been found that the system enjoys an acceptable stability at the time of an earthquake, in order to improve the efficiency and increase the assurance, there is the need to consider a few measures in designing network components and connections from an earthquake engineering point of view.

V. Overall and local instabilities of the electrical grid at the time of an earthquake have been recognized as dominant weak points of a dynamic emergency routes system.

\section{References}

[1] Wikipedia, www.wikipedia.org

[2] Zare M. Personal communication, khabaronline 2012/08/23, www.khabaronline.ir 
[3] Hosseini M., Lifelines and Their Conditions in City of Tehran, Pejouheshnameh (The Persian Journal and Bulletin of IIEES), May 1996.

[4] Hosseini M., Problems of Tehran Metropolis from Urban Planning and Design Point of View and Some Resolving Solutions, Pejouheshnameh (The Persian Journal and Bulletin of IIEES), Vol. 10, No. 1, winter 2007.

[5] Hosseini M., On the Effect of Urban Features on Human Casualty Level in Large and Populated Cities, Asian Disaster Management News, adpc, Vol. 13, No. 2, May - August 2007.

[6] Hosseini M., How much is the readiness of Tehran to earthquakes? first seminar of construction in municipality, 2002.

[7] M. Sadeghi, M. Hosseini, N. Pakdel Lahiji, “Developing Fragility Curves for Seismic Vulnerability Assessment of Tubular Steel Power Transmission Tower Based on Incremental Dynamic Analysis”, 15th World Conference on Earthquake Engineering, Lisbon, Portugal.

[8] Khalije fars news agency, www.pgnews.ir/, 2012/02/08. 